\title{
Avaliação de diferentes protocolos no clareamento dentário
}

\author{
Evaluación de diferentes protocolos en el blanqueamiento dental \\ Evaluation of different protocols in dental bleaching \\ Talina Rocha Balbina dos SANTOS ${ }^{1}$ \\ Rafaelly Galindo dos Santos PEREIRA ${ }^{2}$ \\ Pedro Vinícius de Melo ALVES ${ }^{2}$ \\ Thayse Gonzaga GOMES ${ }^{1}$ \\ Pedro Henrique SETTE-DE-SOUZA \\ ${ }^{1}$ Departamento de Odontologia, UEPB Univ Estadual da Paraíba, 58233-000 Araruna - PB, Brasil \\ ${ }^{2}$ Departamento de Odontologia, UPE Univ de Pernambuco, 56512-200 Arcoverde - PE, Brasil
}

\begin{abstract}
Resumo
Introdução: A estética é a principal busca nos consultórios odontológicos e um dos tratamentos mais realizados é o clareamento dentário, a cada ano mais profissionais buscam a melhor técnica no que diz respeito a longevidade, efetividade, facilidade de passos clínicos e menor desconforto para o paciente Objetivo: Avaliar a influência da modificação do protocolo para aplicação de agentes clareadores utilizados em consultórios odontológicos, determinando a mudança de cor de diferentes agentes clareadores em dentes submetidos a diferentes protocolos. Material e métodos: Uma amostra de 20 dentes selecionados e codificados tiveram suas cores estabelecidas de acordo com a escala Vita Classica. Seccionados e divididos em quatro grupos entre protocolo convencional e modificado e uso dos agentes clareadores Whiteness HP e Whiteness HP Maxx. Resultados: A modificação do protocolo alcança resultados satisfatórios e sem diferença estatisticamente significativa quando comparado ao que é preconizado pelos fabricantes. Conclusões: Os resultados sugerem que a modificação pode representar uma ferramenta útil na prática clínica, uma vez que permite torna-lo mais eficaz, diminuir custos e quantidade de material utilizado.
\end{abstract}

Descritores: Clareamento Dental; Peróxido de Hidrogênio; Clareadores.

\section{Abstract}

Introduction: Aesthetics is the main search in dental offices and one of the most accomplished treatments is dental bleaching, each year more professionals seek the best technique regarding longevity, effectiveness, ease of clinical steps and less discomfort for the patient Objective: To evaluate the influence of the modification of the protocol for the application of bleaching agents used in dental practices, determining the color change of different bleaching agents in teeth submitted to different protocols. Material and methods: A sample of 20 selected and coded teeth had their colors established according to the Vita Classica scale. Sectioned and divided into four groups between conventional and modified protocol and use of whitening agents Whiteness HP and Whiteness HP Maxx. Results: Modification of the protocol achieves satisfactory results and without statistically significant difference when compared to what is recommended by the manufacturers. Conclusions: The results suggest that the modification can represent a useful tool in clinical practice, since it allows to makes it more effective, to reduce costs and quantity of material used.

Descriptors: Tooth Bleaching; Hydrogen Peroxide; Bleaching Agents.

\section{Resumen}

Introducción: La estética es la principal búsqueda en los consultorios odontológicos y uno de los tratamientos más realizados es el blanqueamiento dental, cada año más profesionales buscan la mejor técnica en lo que se refiere a la longevidad, efectividad, facilidad de pasos clínicos y menor incomodidad para el paciente Objetivo: Evaluar la influencia de la modificación del protocolo para aplicación de agentes blanqueadores utilizados en consultorios odontológicos, determinando el cambio de color de diferentes agentes blanqueadores en dientes sometidos a diferentes protocolos. Material y métodos: Una muestra de 20 dientes seleccionados y codificados tuvo sus colores establecidos de acuerdo con la escala Vita Classica. Seccionados y divididos en cuatro grupos entre protocolo convencional y modificado y uso de los agentes blanqueadores Whiteness HP y Whiteness HP Maxx. Resultados: La modificación del protocolo alcanza resultados satisfactorios y sin diferencia estadísticamente significativa en comparación con lo que preconizan los fabricantes. Conclusiones: Los resultados sugieren que la modificación puede representar una herramienta útil en la práctica clínica, ya que permite hacer que sea más eficaz, disminuir los costos y la cantidad de material utilizado.

Descriptores: Blanqueamiento de Dientes; Peróxido de Hidrógeno; Blanqueadores.

\section{INTRODUÇÃO}

O cuidado com a estética não é exclusivo da sociedade contemporânea, já que há relatos de uso de vinagre associado a substâncias abrasivas, pelas civilizações antigas, na esperança de branquear os dentes ${ }^{1}$. A estética é a principal busca nos consultórios odontológicos e tornou-se um grande desafio para os dentistas alcançá-la ${ }^{2 .}$ Um procedimento estético muito realizado no consultório odontológico é o clareamento dentário, e a cada ano mais profissionais buscam a melhor técnica no que diz respeito a longevidade, efetividade, facilidade de passos clínicos e menor desconforto para o paciente ${ }^{3}$ que apresentam manchas amarelas, marrons ou áreas brancas em uma superfície, ou várias, no esmalte ${ }^{2}$. $\mathrm{O}$ tratamento clareador possui algumas características vantajosas em relação aos demais procedimentos estéticos: possui uma técnica minimamente invasiva que é preconizada na Odontologia contemporânea, apresenta baixo custo e o tempo de execução é o menor para a maioria dos casos.

Para o clareamento dos dentes existem métodos diferentes, cada um com seu próprio mecanismo de ação que lhe garante a eficácia a depender do tipo de descoloração a ser tratada ${ }^{4}$. A anamnese e o exame clínico e radiográfico são imprescindíveis para conhecer as aspirações dos pacientes e também detectar a causa do escurecimento dentário ${ }^{3}$. A cor dos dentes é influenciada por uma combinação de cores intrínsecas e extrínsecas devido às manchas que se formam sobre a superfície do dente. As manchas intrínsecas ou internas podem ser atribuídas a fatores congênitos ou adquiridas, como: genética, idade, a partir da exposição da dentina amarelada, antibióticos, altos níveis de fluoretos, falhas nos estágios de erupção, iatrogênicas, necrose pulpar ou 
após restaurações ${ }^{4}$ e as extrínsecas podem ser causadas por corantes alimentares, uso de tabaco, ou acúmulo de placa bacteriana e tártaro dentário ${ }^{2}$.

O paciente deve ser avaliado durante anamnese para identificação dos possíveis fatores de risco, como a presença de trincas, restaurações desadaptadas, lesões cariosas, áreas de recessão gengival ou qualquer solução de continuidade com o tecido dentinário e mesmo determinar o limiar de dor do indivíduo, que o predisponha aos sintomas dolorosos indesejados. O completo conhecimento de todos estes aspectos é decisivo para que a técnica clareadora mais adequada possa ser empregada na tentativa de se clarear os dentes e minimizar os possíveis efeitos colaterais do tratamento ${ }^{3}$.

No mercado odontológico, podemos encontrar uma diversidade de produtos clareadores, que podem ser à base de peróxido de hidrogênio ou peróxido de carbamida, em diferentes concentrações. As soluções de peróxido de carbamida são muito instáveis e dissociam-se facilmente ao entrarem em contato com o tecido dental ou a saliva formando 35\% de peróxido de hidrogênio e 7-10\% de ureia. Posteriormente, o peróxido de hidrogênio se degradará em oxigênio e água, enquanto a ureia se degradará em amônia e dióxido de carbono ${ }^{5,6}$.

Estudos corroboram que o efeito de branqueamento é proporcional à concentração de peróxido de hidrogênio ${ }^{7-9}$. Os géis com concentrações mais altas entrelaçam os dentes a um ritmo mais rápido, sendo mais propensos a causar sensibilidade ${ }^{9}$. No estudo de Gonçalves et al. ${ }^{8}$ foi notado está evidência, na qual o gel com menor concentração, apesar de ter tido o tempo de contato mais longo foi o que causou menor sensibilidade.

O peróxido de hidrogênio é o ingrediente ativo dos géis clareadores: é um forte agente oxidante que possui a capacidade de difundir pelas estruturas dentárias mineralizadas em função da permeabilidade destes substratos e devido ao baixo peso molecular dessas substâncias ${ }^{10}$. O mecanismo de ação dos agentes clareadores é baseado num complexo processo de oxidação.

Das técnicas utilizadas para o tratamento clareador, três se tornaram o objetivo de estudos dos pesquisadores e são utilizados pelos profissionais da área: o clareamento caseiro, o de consultório e o uso de produtos autoadministráveis. O caseiro consiste no uso de um agente clareador de baixa concentração em moldeira de silicone, sob prescrição do cirurgião-dentista da quantidade e o período de tempo diário. Esta terapia permite um aprimoramento de cor dental clinicamente perceptível mesmo depois uma aplicação clínica $^{11}$.

Enquanto as aplicações em consultório utilizam-se um peróxido em altas concentrações (25-35\%), realizada a barreira gengival, aplica-se o gel clareador diretamente nos dentes, pode ser ativado por luz ou não. Os produtos autoadministráveis são comercializados como dentifrícios, goma de mascar, tiras adesivas clareadoras, vernizes, e contém concentrações de agentes clareadores entre $3 \%$ a $6 \%$ de peróxido de hidrogênio ${ }^{6}$.

Entre as desvantagens, quando o peróxido entra em contato repentino com a gengiva, a sensibilidade dos dentes e a irritação gengival são relatados frequentemente pelos pacientes submetidos a terapias de clareamento dentário ${ }^{12}$. Acredita ser consequentemente, pelo menos em parte, da difusão do Peróxido de hidrogênio através do esmalte e dentina para dentro da câmara pulpar. Este fenómeno resulta em inflamação da polpa, com libertação de mediadores inflamatórios e estímulo nervos sensoriais ${ }^{11}$. Em alguns casos, a sensibilidade é tão intensa que o tratamento é interrompido.

Diante do exposto, o objetivo deste trabalho foi avaliar a influência da modificação do protocolo para aplicação de agentes clareadores utilizados em consultórios odontológicos, determinando a mudança de cor de diferentes agentes clareadores em dentes submetidos a diferentes protocolos.

\section{MATERIAL E MÉTODO}

Por se tratar de uma pesquisa que utilizará material biológico, a pesquisa foi aprovada pelo Comitê de Ética em Pesquisa da UEPB sob número 62510116.0.0000.5187. Através de uma parceria, os dentes para a pesquisa foram doados pelo Banco de Dentes Humanos do curso de Odontologia da UEPB Campus VIII, conforme preconizado pela resolução do Conselho Nacional de Saúde 466/2012. Para essa finalidade, apenas os dentes com coroa hígida foram selecionados. Para o preparo dos blocos dentários e seleção dos grupos, foram feitas a identificação e registro inicial da cor dos dentes. Os dentes tiveram sua cor estabelecida de acordo com a escala VITA® clássica (Zahnfabrik, Sackingen, Alemanha), com metodologia proposta por Polydorou et al. ${ }^{13}$ a qual se organiza a escala não pelo matiz, mas sim pelo valor da cor, e cada cor recebe um escore $(\mathrm{B} 1=1, \mathrm{~A} 1=2$, $\mathrm{B} 2=3 \ldots)$.

Para selecionar a cor, dois examinadores independentes passaram por um processo de calibração prévia. Após término do processo de calibração, os examinadores estabeleceram a cor dos dentes. A cada cinco seleções feita, os examinadores fizeram o re-teste, para garantir a padronização. Cada examinador registrou a cor de todos os 20 dentes da amostra, de maneira independente. Os dentes foram seccionados na junção amelocementária por um disco de diamante para separar a porção coronária da radicular. Após esse procedimento, a porção coronária foi seccionada com o mesmo disco no sentido vestíbulo-lingual (corte sagital), para separar o dente em duas porções, mesial e distal. Se a porção 
mesial de um bloco ficou no grupo 1 ou 3, a porção distal do mesmo ficou obrigatoriamente no grupo 2 ou 4. A distribuição em cada grupo foi sequencial. Ao término do processo de amostragem, cada grupo teve 10 blocos.

- Grupo 1 = Protocolo convencional - Whiteness HP

- Grupo 2 = Protocolo modificado - Whiteness HP

- Grupo 3 = Protocolo convencional - Whiteness HP Maxx

- Grupo 4 = Protocolo modificado - Whiteness HP Maxx

Para o estudo, levaram-se em conta os géis clareadores a base de peróxido de Hidrogênio na concentração 35\% da FGM (Whiteness HP Maxx e Whiteness HP) para ver se há diferença no clareamento entre eles.

O protocolo dos grupos 1 e 3 foram preconizados pelo fabricante, com a retirada do gel clareador a cada 15 minutos, seguido de nova aplicação, totalizando três aplicações ao final do ciclo. O protocolo dos grupos 2 e 4 tiveram como modificação a não retirada do gel clareador. Para esses grupos, eventuais movimentações do gel sobre a superfície dentária foram realizadas a cada cinco minutos. O tempo de exposição total do gel sobre o dente foi padronizado conforme preconiza $o$ fabricante (45 minutos). Os ensaios foram realizados em duas sessões, distantes entre si oito dias. Após o período de clareamento, a cor foi novamente registrada. A variação da cor foi obtida pela diferença entre a cor final e a cor inicial do bloco.

Para avaliar a concordância intra e interexaminador durante a calibração e seleção de cor, foi feito o teste estatístico para avaliar a significância do Kappa. Para comparação da alteração de cor entre os grupos foi utilizado o teste de comparações múltiplas através da Análise de Variância (ANOVA).

\section{RESULTADOS}

Como os dados não apresentaram distribuição normal foi realizado o teste de KruskallWallis para verificar se havia diferença estatisticamente significativa entre os grupos. Constatada tal diferença, lançou-se mão do teste de Mann-Whitney com penalização de Bonferroni para verificar entre quais grupos estava essa diferença.

Tabela 1: Mediana (Quartil 25 / Quartil 75) da cor inicial, final e resultado do tratamento (após 7 dias). Letras iguais indicam ausência de diferença estatisticamente significativa.

\begin{tabular}{ccccc}
\hline Produto & Cor Inicial & Cor Final & Diferença & $\begin{array}{c}\text { p } \\
\text { valor }\end{array}$ \\
\hline HP 35 3 $\mathrm{x}^{\mathrm{a}}$ & $6,75(5,12 / 8,87)$ & $1,25(1,00 / 1,50)$ & $5,25(4,00 / 7,12)$ & \\
HP 35 $1 \mathrm{x}^{\mathrm{a}} \mathrm{b}$ & $6,75(5,12 / 8,87)$ & $4,00(3,12 / 4,37)$ & $4,25(1,12 / 5,00)$ & 0,048 \\
HPMax 3 $\mathrm{x}^{\mathrm{b}}$ & $5,75(5,12 / 7,00)$ & $4,00(3,00 / 5,00)$ & $2,00(1,50 / 3,37)$ & \\
${\text { HPMaxx } 1 \mathrm{x}^{\mathrm{a}, \mathrm{b}}}$ & $5,75(5,12 / 7,00)$ & $1,75(1,00 / 4,50)$ & $4,25(2,62 / 4,87)$ & \\
\hline
\end{tabular}

A modificação do protocolo alcança resultados satisfatórios e sem diferença estatisticamente significativa quando comparado ao que é preconizado pelos fabricantes.
DISCUSSÃO

O processo de clareamento é possível devido ao baixo peso molecular do peróxido de hidrogênio que permite a sua penetração no esmalte e dentina ${ }^{13,14}$ formando radicais livres, moléculas reativas de oxigênio, que atacam as moléculas escuras, quebrando-as em moléculas menores e mais claras $^{15,16 .}$.

Uma das maiores dificuldades de estudos sobre clareamento dental é mensurar o efeito clareador. Fotografias intra-bucais, comparação com escalas de cor e o emprego de espectrofotômetro ou colorímetro têm sido utilizados ${ }^{17,18}$. Contudo, ainda é difícil comparar estudos que avaliaram a eficácia do clareamento devido a grande diferença no tamanho das amostras, nos produtos clareadores, no número de sessões de clareamento, nos dentes considerados para análise, na duração do controle após o tratamento, além da grande variedade de técnicas.

Neste estudo, fez-se a comparação com a escala de cor VITA® clássica, que é o método mais usado na prática clínica diária, por ser rápido e simples tem sido utilizado com sucesso em muitos estudos, oferecendo resultados clinicamente relevantes, porque um clareamento efetivo implica em alteração de cor visualmente perceptível ${ }^{13,17}$. Dessa forma, a alteração cromática não teve diferença estatisticamente significativa, indicando que de fato os diferentes protocolos utilizados promoveram um grau de mudança de cor semelhante. Estes dados mostram que o tratamento clareador independe do protocolo utilizado.

A busca por um procedimento mais barato também impulsiona a procura, e diminuindo a quantidade de peróxido utilizada há a possibilidade de diminuir os custos e garantir benefícios. Soares et al. ${ }^{11}$ mostraram que a penetração excessiva de peróxidos no complexo dentino-pulpar é preocupante pois podem ocasionar injúrias ao tecido pulpar devido a difusão do peróxido de hidrogênio através do esmalte e dentina para o interior da câmara pulpar $^{10} \mathrm{e}$, portanto, modificar o protocolo utilizado pode diminuir também a sensibilidade e injúrias a polpa.

O desenvolvimento de novas técnicas parece ter sido impulsionado pelos fabricantes de equipamentos de branqueamento de energia $\mathrm{e}$ materiais em vez de exigir uma abordagem baseada em evidências ${ }^{19}$.

Por ser um estudo in vitro, estes resultados não podem ser extrapolados diretamente para uma situação clínica ${ }^{20}$. Embora os resultados do presente estudo possam ter relevância clínica, existem algumas diferenças em comparação a reais condições. Em primeiro lugar, a utilização de dentes extraídos provavelmente permite uma penetração mais rápida do agente de branqueamento para a polpa, uma vez que eles eram desprovidas do fluido de dentina 
produzida por pressão intrapulpar ${ }^{21-25}$. Segundo, a ausência de tecidos celulares podem também ter alterado a absorção dos padrões de subprodutos ${ }^{20}$. Soma-se a isso, o fato do tecido pulpar apresentar sistemas linfáticos e antioxidantes que protegem as células pulpares contra agressões externas ${ }^{25,26}$. Ainda assim, futuros estudos deverão ser conduzidos para elucidar os mecanismos celulares envolvidos na resposta biológica ao clareamento dentário, o que permitirá eliminar ou mesmo minimizar estes efeitos $^{27}$.

Atualmente o tratamento clareador de dentes vitais pode ser considerado não unicamente um procedimento estético, mas uma terapia de fato, em que um medicamento é aplicado na superfície vestibular de dentes, o que comprova a necessidade de estabelecer-se a relação tempo/concentração para uma posologia individualizada. Os resultados obtidos no presente estudo questionam a o tempo de contato do peróxido nos dentes para obter efeito clareador. E indica que os dentistas devem basear suas decisões sobre o tratamento também em evidências científicas e não apenas nas orientações dos fabricantes destinadas ao clareamento dental.

\section{CONCLUSÃO}

$\mathrm{Na}$ análise dos índices é perceptível a importância de que os dentistas devem basear suas decisões sobre o tratamento também em evidências científicas e não apenas nas orientações dos fabricantes destinadas ao clareamento dental. Portanto, achados clínicos somados as evidências estudadas devem embasar em melhor desempenho do protocolo escolhido.

Além disso, os resultados sugerem que a modificação pode representar uma ferramenta útil na prática clínica, uma vez que permite torná-lo mais eficaz, diminuir custos e quantidade de material utilizado. Consequentemente gerando menos efeitos colaterais pós-clareamento dentário, evitando também o uso de terapias medicamentosas.

\section{REFERÊNCIAS}

1. Souza LCM, Rodrigues CRT, Pereira VFGC, Oliveira Júnior NG. Analysis of stability of color resin composite under three concentrations carbamide peroxide. J Surg Clin Dent. 2014;3(1):05-11.

2. Sundfeld RH, Sundfeld Neto D, Machado LS, Franco LM, Fagundes TC, Briso ALF. Microabrasion in tooth enamel discoloration defects: three cases with long-term follow ups. J Appl Oral Sci. 2014; 22(4):347-54.

3. Briso ALF, Rahal V, Gallinari MO, Moreira JC, Almeida LCAG, Mestrener LR. Análise do clareamento dental caseiro realizado com diferentes produtos - relato de caso. Rev Odontol Araçatuba. 2014; 35(1):49-54.
4. Carey CM. Tooth whitening: what we now know. J Evid Based Dent Pract. 2014; 14(Suppl):70-6.

5. Silva FMM, Nacano LG, Gava Pizi EC. Clinical Evaluation of two tooth whitening Systems. Rev Odontol Bras Central. 2012; 21(56):473-79.

6. Yamamoto TW. Efeito da utilização de dentifrícios com diferentes compostos bloativos nas propriedades superficiais do esmalte dental clareado [dissertação]. São Paulo: Universidade de São Paulo USP, Faculdade de Odontologia; 2012.

7. Llambes G, Llena C, Amengual J, Forner L. In vitro evaluation of the efficacy of two bleaching procedures. Med Oral Patol Oral Cir Bucal. 2011; 16(6):e845-51.

8. Gonçalves MLL, Tavares ACDS, Mota ACCD, Penna LAP, Deana AM, Busadori SK. In-office tooth bleaching for adolescents using hydrogen peroxide-based gels: clinical trial. Braz Dent J. 2017; 28(6):720-25.

9. Machado LS, Oliveira FG, Rocha EP, dos Santos $\mathrm{PH}$, Briso ALF, Sundefeld MLMM et al. Clinical trial evaluating color change and tooth sensitivity throughout and following in-office bleaching. Int J Periodontics Restorative Dent. 2013; 33(2):209-15.

10.Cintra LTA, Benetti F, Ferreira LL, Rahal V, Ervolino E, Jacinto RC et al. Evaluation of an experimental rat model for comparative studies of bleaching agents. J Appl Oral Sci. 2016; 24(1):95-104.

11. Soares DG, Basso FG, Hebling J, de Souza Costa CA. et al. Concentrations of and application protocols for hydrogen peroxide bleaching gels: effects on pulp cell viability and whitening efficacy. J Dent. 2014; 42(2):185-98.

12.Kim JH. Oral composition for tooth whitening product, and kit compressing same. LG Household \& Health Care Ltd. Seoul: 2018.

13.Polydorou O, Wirsching M, Wokewitz M, Hahn P. Three-month evolution of vital tooth bleaching using light units: A randomized clinical study. Oper Dent. 2013; 38(1):21-32.

14.Luk K, Tam L, Hubert M. Effect of light energy on peroxide tooth bleaching. J Am Dent Assoc. 2004; 135(2):194-201.

15.Kawamoto K, Tsujimoto Y. Effects of the hydroxyl radical and hydrogen peroxide on tooth bleaching. J Endod. 2004; 30(1):45-50

16. Yazici AR, Khanbodaghi A, Kugel G. Effects of an in-office bleaching system on pulp chanber temperature in vitro. J. Contemp Dent Pract. 2007; 8(4):19-26.

17. Marson FC, Sensi LG, Vieira LC, Araújo E. Clinical evaluation of in-office dental bleaching treatments with and without the use of lightactivations sources. Oper Dent. 2008;33(1):15-22. 
18.dos Santos Medeiros MC, de Lima KC. Effectiveness of night guard vital bleaching with 10\% carbamide peroxide - a clinical study. J Can Dent Assoc. 2008; 74(2):163-163e.

19.Patel A, Louca C, Millar BJ. An in vitro comparison of tooth whitening techniques on natural tooth colour. $\mathrm{Br}$ Dental J. 2007; 204(9):E15.

20.Gonçalves RS. Efeito de diferentes fontes luminosas e pré-condicionamento do esmalte na alteração de cor, penetração de $\mathrm{H}_{2} \mathrm{O}_{2}$ e citotoxicidade em dentes clareados [dissertação]. Araçatuba: Univ Estadual Paulista (UNESP), Faculdade de Odontologia; 2014.

21.Hanks CT, Fat JC, Wataha JC, Corcoran JF. Cytotoxicity and dentin permeability of carbamide peroxide and hydrogen peroxide vital bleaching materials, in vitro. J Dent Res. 1993; 72(5):931-38

22. Sulieman M, Addy M, Rees JS. Development and evaluation of a method in vitro to study the effectiveness of tooth bleaching. J Dent. 2003; 31(6):415-22.

23.Zimmerman B, Datko L, Cupelli M, Alapati S, Dean D, Kennedy M. Alteration of dentin-enamel mechanical properties due to dental whitening treatments. J Mech Behav Biomed Mater. 2010; 3(4):339-46

24.Buzalaf MA, Hannas AR, Kato MT. Saliva and dental erosion. J App Oral Sci. 2012; 20(5):493502

25.Kina JF, Huck C, Riehl H, Martinez TC, Sacono NT, Ribeiro AP et al. Response of human pulps after professionally applied vital tooth bleaching. Int Endod J. 2010; 43(7):572-80

26. Sauro S, Pahley DH, Montanari M, Chersoni S, Carvalho RM, Toledano $M$ et al. Effect of simulated pulpal pressure on dentin permeability and adhesive of self-etch adhesives. Dent Mat. 2007; 23(6):705-13

27. Almeida LCEA, Riehl H, Santos PH, Sundfeld ML, Briso AL. Clinical evaluation of the effectiveness of different bleaching therapics in vital teeth. Int J Peridontics Restorative Dent. 2012; 32(3):303-9.
CONFLITO DE INTERESSES

Os autores declaram não haver conflitos de interesse.

\section{AUTOR PARA CORRESPONDENCIA}

\section{Pedro Henrique Sette de Souza}

pedro.souza@upe.br

Submetido em 09/08/2018

Aceito em 04/10/2018 